silencing by pairing and heterochromatin formation ${ }^{8}$, and it has also been shown that DNA methylation and supercoiling stress can influence the likelihood of the formation of alternative non-B DNA $^{9,10}$. Since all the repeats identified in Table 1 are in regions subject to mono-parental methylation, it is possible that methylation could preferentially influence the local DNA structure on one of the parental chromosomes. Keeping this in mind,

1. Michaud, E.J. et al. Genes Dev. 8, 1463-1472 (1994).

2. Duhl, D.M.J., Vrieling, H., Miller, K.A., Wolff, G.L. \& Barsh, G. S. Nature Genet. 8, 59-65 (1994).

3. Barlow, D.P. Trends Genet. 10, 194-198 (1994).

4. Barlow, D.P. Science 280, 309-310 (1993)

5. Stoger, R. et al. Cell 73, 61-71 (1993).

6. Larsen, F., Gunderson, G., Lopez, R. \& Prydz, H. one of the many models that could be envisaged isonewhere transcriptionally induced supercoiling stress, in repeat containing regions, could induce the formation of non-BDNAand influence the processivity of the transcription machinery. The role of mono-parental methylation would be to restrict these effects to one of the parental chromosomes.

Taken together, it seems quite tempting to concentrate the search for new imprinted genes on genomic

Genomics 13, 1095-1107 (1992).

7. Hastle, N. D. pp 559-574. in Genetic variants and strains of the laboratory mouse. Eds. Lyon, M.F. and Searle, A. OUP. Oxford. UK. (1989).

8. Dorer, D.R. and Henikoff, S. Cell 77, 993-1002 (1994).

9. Zacharias, W. pp 27-38 in DNA methylation: Molecular Biology and Biological Significance (eds. Jost, J.P. and Saluz, H.P.) (Birkhăuser segments with the following characteristics; CG rich, monoparental methylation and the presence of direct repeats. With a little bit of luck, an imprinted gene may be close by.

\section{Bjorn Neumann \\ Pavel Kubicka}

Denise P. Barlow

Research Institute for Molecular Pathology (I.M.P.), Dr. Bohr-Gasse 7, A-1030 Vienna, Austria

Verlag, Basel., 1993).

10. van Holde, $K$, and Zlatanova, J. BioEssays 16 59-68 (1993).

11. Efstratiadis, A. Curr. Opin. Genet. Dev. 4, 265280 (1994).

12. Sutcliffe, J. S. et al. Nature Genet. 8, 52-58 (1994).

13. Kuff, E.L. \& Lueders, K.K. Adv. in Cancer Rec. 51, 183-276 (1988).

\title{
Genetic analysis of complex disease
}

Sir - We appreciated much of what Glenys Thomson writes in the October 1994 issue of Nature Genetics regarding appropriate methods to detect genetic contributions to complex diseases, using the example of the recent progress in understanding the genetics of diabetes ${ }^{1}$. However, we feel that her emphasis on the use of nuclear families containing affected sib pairs may have the undesirable consequence of discouraging researchers from looking for larger pedigrees with multiple affected members.

Although the affected sib-pair method of analysis is simple and avoids some problems associated with lod score analysis, it has reduced power to detect linkage when applied to larger pedigrees. Moreover, affected sib pairs can provide little information for the mapping of disease locibecause in affected sib pairs recombination, penetrance and admixture are all strongly confounded with each other and it is impossible to estimate one parameter without providing values for the others. Nuclear families can provide a little more information, but not much. With larger pedigrees it becomes much easier to estimate the parameters of the transmission model and to assess the contribution and likely position of the disease gene. Methods have now been described which can detect linkage in extended pedigrees as well as sib pairs without needing to make any assumptions concerning themodeof transmission ${ }^{2,3}$, and it is also possible to modify the lod score method so it can be applied in situations where the mode of transmission is unknown, as was demonstrated in the paper by Field and colleagues. In our own simulations, we have found that even when the mode of transmission is unknown pedigrees can yield far more information for the detection oflinkage than sib pairs containing the same number of affected subjects (submitted for publication).
As methods suitable for the analysis of complex diseases are available for pedigrees of any structure, and as larger pedigrees yield far more information for detecting linkage and for mapping loci than small ones, we urge researchers investigating complex diseases to take every opportunity to augment their samples by extending pedigrees wherever more distant affected relatives can be found rather than focussing exclusively on nuclear families.

\section{David Curtis}

Pak C. Sham

Homero P. Vallada

Departments of Psychological Medicine and ${ }^{1}$ Biostatistics, Institute of Psychiatry, De Crespigny Park, London SE5 8AF UK

\footnotetext{
1. Thomson, G. Nature Genet. 8, 108-110 (1994).

2. Weeks, D.E. \& Lange, K. Am. J. hum. Genet. 42 315-326 (1988).

3. Curtis, D. \& Sham P.C. Ann. hum. Genet. 58, 151-162 (1994)

4. Fleld, L.L., Tobias, R. \& Magnus, T. Nature
} Genet. 8,189-194 (1994). 